Purdue University

Purdue e-Pubs

2012

\title{
Direct Simulation of Thermal Transport through Sintered Wick Microstructures
}

\author{
K. K. Bodla \\ Purdue University \\ J. Y. Murthy \\ S V. Garimella \\ Purdue University, sureshg@purdue.edu
}

Follow this and additional works at: http://docs.lib.purdue.edu/coolingpubs

Bodla, K. K.; Murthy, J. Y.; and Garimella, S V., "Direct Simulation of Thermal Transport through Sintered Wick Microstructures" (2012). CTRC Research Publications. Paper 214.

http://dx.doi.org/10.1115/1.4004804

This document has been made available through Purdue e-Pubs, a service of the Purdue University Libraries. Please contact epubs@purdue.edu for additional information. 


\section{Direct Simulation of Thermal Transport Through Sintered Wick Microstructures}

\section{Karthik K. Bodla} Jayathi Y. Murthy

\section{Suresh V. Garimella}

\author{
Cooling Technologies Research Center, \\ an NSF IUCRC, \\ School of Mechanical Engineering and \\ Birck Nanotechnology Center, \\ Purdue University, \\ West Lafayette, IN 47907-2088
}

Porous sintered microstructures are critical to the functioning of passive heat transport devices such as heat pipes. The topology and microstructure of the porous wick play a crucial role in determining the thermal performance of such devices. Three sintered copper wick samples employed in commercial heat pipes are characterized in this work in terms of their thermal transport properties_-porosity, effective thermal conductivity, permeability, and interfacial heat transfer coefficient. The commercially available samples of nearly identical porosities ( $21 \%$ open volume) are CT scanned at $5.5 \mu \mathrm{m}$ resolution, and the resulting image stack is reconstructed to produce high-quality finite volume meshes representing the solid and interstitial pore regions, with a conformal mesh at the interface separating these two regions. The resulting mesh is then employed for numerical analysis of thermal transport through fluid-saturated porous sintered beds. Multiple realizations are employed for statistically averaging out the randomness exhibited by the samples under consideration. The effective thermal conductivity and permeability data are compared with analytical models developed for spherical particle beds. The dependence of effective thermal conductivity of sintered samples on the extent of sintering is quantified. The interfacial heat transfer coefficient is compared against a correlation from the literature based on experimental data obtained with spherical particle beds. A modified correlation is proposed to match the results obtained. [DOI: $10.1115 / 1.4004804]$

Keywords: porous media, effective thermal conductivity, permeability, microtomography, sintered beds, heat pipes

\section{Introduction}

Sintered copper is an important engineering material which finds use in applications as diverse as brake pads and heat pipes. Heat pipes and vapor chambers are passive, two-phase heat transport devices capable of transporting heat over long distances without a substantial drop in temperature $[1,2]$. Heat pipes are fabricated in several common configurations and consist of sealed chambers containing a working fluid such as water, which transports heat through phase change. These devices exploit wicking due to capillary action generated by the wicking material lining the inside, which enables them to passively transport the working fluid. With effective thermal conductivities that are two or three orders of magnitude higher than for solid metal structures of the same dimensions, these devices find widespread application in the field of thermal management [3]. Some of the specific applications of such two-phase heat transport devices in the electronics cooling industry are hot spot cooling and heat spreading [4].

The wick and the working fluid are the two most important components of a heat pipe. Water is the most commonly employed working fluid in the low temperature range necessary for electronics cooling [1]. It has desirable thermophysical properties such as heat of vaporization and surface tension, in addition to being safe to use. Wicks, on the other hand, can be of many different kinds and are made of a variety of materials. Some of the common wick types are: homogeneous wicks, such as those made of wrapped screen and sintered metal, and composite wicks, for example, composite screen wicks and screen-covered groove wicks. Composite wicks provide high capillary pressure and high permeability but are more difficult to manufacture. The high production cost of composite

Contributed by Heat Transfer Division of ASME for publication in the JouRnAL OF Heat Transfer. Manuscript received January 17, 2011; final manuscript received July 27, 2011; published online November 29, 2011. Assoc. Editor: Andrey Kuznetsov. wicks limits them to specific applications. Homogeneous wicks, on the other hand, are inexpensive and are widely employed.

The most common homogeneous wick configuration is the screen mesh. It consists of a metal fabric placed on the inside of the heat pipe casing. The rectangular pores in the woven fabric generate the capillary pressure necessary to sustain fluid transport. Some of the limitations of this wick are its considerably lower capillary pressure (compared, for example, to sintered wicks) and low effective thermal conductivity. Porous sintered metal wicks overcome some of these shortcomings. Copper is a commonly employed sintered metal wicking material because of its high thermal conductivity and compatibility with water.

Estimation of the transport properties of wick structures is essential for designing high-performance heat pipes. Early thermal analyses of porous beds have been largely experimental [5]. Porosity or void fraction can be estimated by a number of experimental methods such as imbibition and direct crushing. Dullien [5] provided an excellent review of the various experimental measurement techniques commonly employed. Some of these methods are known to give an exact porosity (such as the destructive crushing technique), while others such as imbibition yield an effective value, i.e., porosity of the interconnected pores. Most recently Lin et al. [6] measured the porosity of monoporous sintered copper using the imbibition technique.

Effective thermal conductivity, i.e., the thermal conductivity of the sample considering conduction through both the metal and the static fluid, is estimated experimentally by measuring the temperature difference and heat flux in the direction of heat conduction. Generally, owing to experimental limitations such as minimizing the amount of heat loss in the lateral directions through insulation, as pointed out by Lin et al. [6], the uncertainty in measuring effective thermal conductivity is quite high and researchers tend to employ simple analytical models. Some of the analytical models developed for spherical particle beds are the Maxwell-Eucken 
models [7], initially developed for electrical conduction and extended to thermal conduction by analogy. Other models include the effective medium theory model [8,9], which are found to be better suited for heterogeneous, random porous media. Recently Carson et al. [10] compared and contrasted the various thermal conductivity models available in the literature in terms of the domain of applicability, viz., open porosity structures versus closed porosity structures. Maxwell-Eucken models, developed analytically by considering spherical particles to be far apart so as to not influence the other particles, serve as the theoretical bounds for predicting the effective thermal conductivity of porous media. Also, most of these models are developed with porosity as the dominant factor influencing effective thermal conductivity, while, as our results demonstrate, for sintered microstructures it is important to develop robust models incorporating the effect of the amount of sintering in addition to porosity.

Transport properties such as permeability are measured by employing Darcy's law and its modified forms such as those developed by Ergun and Orning [11] and Macdonald et al. [12]. Darcy's law is an empirical relationship between pressure drop and fluid flow rate through the porous sample [13]. Some of the other methods for estimation of permeability include employment of capillary models, i.e., representing the porous media as a series of conduits in series, parallel, or other arrangements [14]. The other single-phase heat transfer parameter of interest is the interfacial heat transfer coefficient, which describes the effectiveness of the porous medium for convective heat transfer. Experimentally, it is a challenging parameter to measure, and as pointed out by Kaviany [14], it is often determined indirectly by employing suitable models developed based on volume averaging of the transport equations. Wakao and Kaguei [15] compiled and compared results from various transient and steady-state experiments pertaining to spherical particle beds. Because of the difficulties in measuring this parameter, large experimental uncertainties are incurred, especially at low Reynolds numbers [14].

Numerical modeling of porous media offers a means to overcome some of the challenges encountered in experimental analysis. Sintered geometries are often represented as spherical packing in a periodic arrangement and thereby the properties are estimated by considering periodic unit cells. Some of the geometries commonly employed for representing sintered microstructures are the BCC and HCP [16] arrangements. Some of the more novel and realistic approaches include employing nondestructive visualization techniques that are beginning to emerge as reliable alternatives for experimental analysis. In our earlier work [17], we demonstrated the applicability of such a technique to predict transport properties of open-cell aluminum foams. In this approach, the numerical model of the porous medium is represented accurately based on the true microstructure. With the advent of superior micro computed tomography $(\mu-\mathrm{CT})$ techniques and submicron resolution, it is now possible to visualize intricate details of random porous media. Tabor et al. [18] presented a voxel-based reconstruction method for generating finite volume meshes based on $\mu$-CT data. The algorithm is implemented in a commercial package, SIMPLEWARE [19], which is employed here.

The present work focuses on the characterization of singlephase thermal and fluid transport through porous sintered microstructures using direct simulation based on $\mu$-CT. Properties such as effective thermal conductivity and permeability, which play an important role in evaluating two of the more prevalent heat transfer limitations in heat pipes, viz., the capillary and boiling limits, are estimated numerically. These otherwise difficult-to-measure parameters are estimated by considering three commercially produced sintered copper wick samples. The scanned geometry is subdivided, and the properties estimated on individual subsamples are reported as averaged values to statistically average out geometric randomness. The strong dependence of effective thermal conductivity on the extent of sintering is demonstrated, and correlations are proposed based on the results obtained to predict convective heat transfer through porous sintered beds.

\section{Mesh Generation}

2.1 Sample Preparation. Accurate pore-scale microscopic modeling of porous media is computationally very expensive, which limits the sample sizes that can be analyzed using this technique. Moreover, modeling real porous media is challenging, owing to the high degree of randomness and irregularities exhibited. In this work, we employ $\mu-\mathrm{CT}$ as a tool to capture these intricate features. Samples that are approximately $10 \mathrm{~mm} \times 5 \mathrm{~mm} \times 1$ to $2 \mathrm{~mm}$ in size are carefully cut from the original sintered copper samples integrated onto solid copper substrates using electric discharge machining (EDM), which ensures that the cuts are clean and that no distortions are introduced. The samples thus prepared are imaged with axial $\mu$-CT at 5.5 micron resolution by Microphotonics Inc. using a Skyscan X-ray scanner. The resolution is selected such that the individual sintered particles are well represented in the resulting scan images. Figure 1 shows a sintered copper sample along with a scan image and the region of interest corresponding to the sintered bed. Also shown in Fig. 1 are sample scan images corresponding to the other two particle-size ranges considered in this study.

2.2 Image Processing and Segmentation. Commercial CTscanners offer software packages which employ the complex reconstruction algorithms described previously to generate a stack of images corresponding to the 3D object being scanned, which can be later used for surface/volume reconstruction. However, the resulting images suffer from a number of artifacts which must be rectified before generating CFD meshes. In this work, we employ the image processing and mesh-generating package SIMPLEWARE [19] for this purpose. The procedure is described only briefly here; details may be found in Refs. [17] and [19]. The software has many features such as noise removal, region identification based on grayscale values, and 3D surface/volume reconstruction, after employing which the processed data can be exported in many standard formats for CAD or mesh generation. The "metal artifact reduction filter" is employed to sharpen the original scanned images using parameters as described in Ref. [19]. The sharpened images can now be employed for region identification and segmentation based on grayscale value. X-ray scanners work on the principle of differential absorptivity of different materials to $\mathrm{X}$-rays. The metal is much denser than the surrounding pore region, which is filled with air. The metal absorbs X-rays, while the pore region lets the $\mathrm{X}$-rays pass through. This difference is reflected in the reconstructed image stack, where the brighter regions correspond to the metal and the darker regions to the surrounding pore. But this transition is not crisp, and hence the segmentation is based on a threshold value selected to ensure that the porosity of the reconstructed sample is the same as the nominal porosity provided by the sintered material manufacturer. It may be noted that even though the segmentation is performed to ensure that the porosity matches the quoted value, it is bound to change in the mesh generation step described below, albeit only a little. Some of the particles in the samples under consideration were found to be hollow, and hence the void space is also identified separately. This void fraction (within the solid copper spheres) is found to be below $1 \%$, and the porosity values provided here include void space as part of pore space, unless otherwise stated.

The identified regions are then "stitched" with unit pixel separation between every pair of adjacent images in the $2 \mathrm{D}$ image stack to generate $3 \mathrm{D}$ volumes corresponding to the metal and void domains of the original scanned sample. After this, the model is checked to ensure that there are no unconnected regions or islands; those that exist are removed using the "floodfill" segmentation tool. At this point, the processed data are analyzed for the number of pixels contained, which is directly related to the number of volumes in the finite-volume mesh. A large number of pixels not only increase the number of cells in the mesh, but also the demand on memory in the mesh generation step. Careful downsampling is hence performed while ensuring that the sintered 


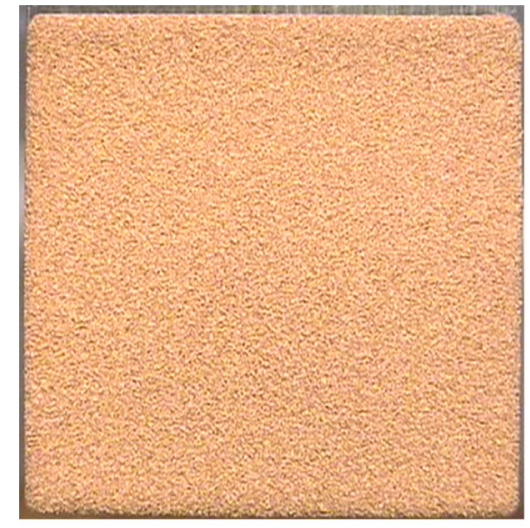

(a)

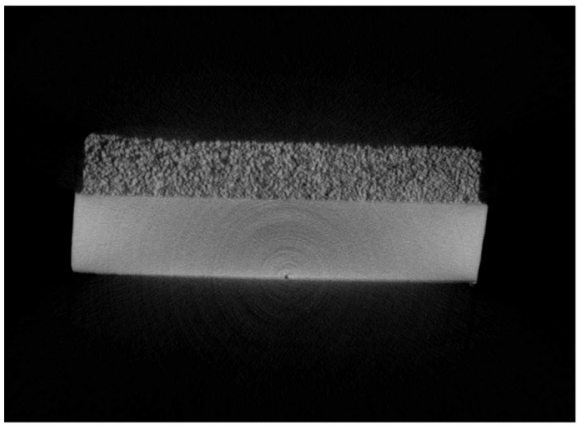

(c)

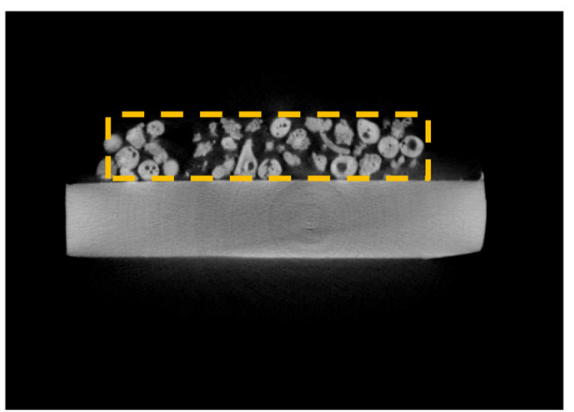

(b)

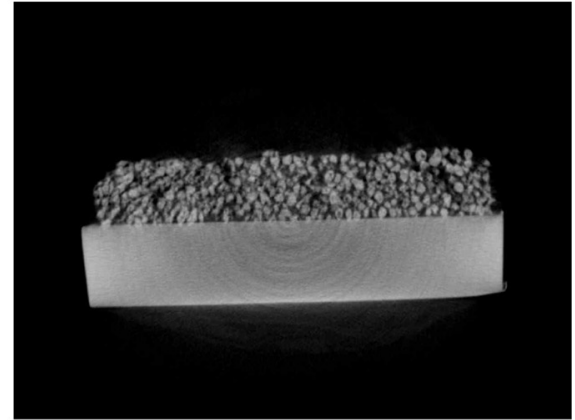

(d)

Fig. 1 (a) Top view of a 250-355 $\mu \mathrm{m}$ sample, (b) scan image in side view, showing the substrate and the sintered regions (sintered region highlighted). Scan images for (c) a 45-75 $\mu \mathrm{m}$ sample and (d) a 106-150 $\mu \mathrm{m}$ sample are also shown.

particles are still well-represented. The number of particles/pores in the scanned data is too large for meshing and subsequent CFD simulations. Consequently the scanned domain is divided into three to four subvolumes, each with 15-35 particles along the longest dimension, and these subvolumes are meshed separately. The results reported in this work are averaged over these subsamples, unless otherwise stated. The various image processing steps are illustrated in Fig. 2 along with the identified metal, pore, and void regions.

2.3 Mesh Generation. The processed subsamples are meshed using the robust "ScanFE-Grid" meshing algorithm in SIMPLEWARE [19], with options such as volumetric and boundary mesh adaptation to optimize and further reduce the number of cells in the mesh. Also, the package provides an option of exporting meshes consisting of either pure tetrahedral or both tetrahedral and hexahedral cells. In this work we employ the latter mesh, with internal and boundary adaption as well as additional smoothing according to the options provided [19]. The smoothed conformal meshes thus generated are of very high quality and consist of approximately $20 \times 10^{6}$ cells for each subvolume (both metal and pore). The mesh so produced is exported to the FLUENT solver [20], which is used for flow and heat transfer analysis. The metal and pore regions, and an inset demonstrating the mesh, are shown in Fig. 3.

\section{Numerical Modeling}

3.1 Governing Equations and Boundary Conditions. The continuity, momentum, and energy equations for the steady, constant-property flow of an incompressible Newtonian fluid are given by

$$
\frac{\partial}{\partial x_{i}}\left(\rho u_{i}\right)=0
$$

$$
\begin{gathered}
\frac{\partial}{\partial x_{j}}\left(\rho u_{j} u_{i}\right)=-\frac{\partial P}{\partial x_{i}}+\mu\left(\frac{\partial^{2} u_{i}}{\partial x_{j} x_{j}}\right) \\
\frac{\partial}{\partial x_{i}}\left(\rho C u_{i} T\right)=\frac{\partial}{\partial x_{i}}\left(k \frac{\partial T}{\partial x_{i}}\right)
\end{gathered}
$$

The CFD computations are performed in the cuboidal computational domain shown in Fig. 3. We perform two types of computations: (a) thermal conduction in the presence of an interstitial fluid, i.e., conduction through both the metal and pore regions and (b) permeability and convective transport through the pore space. In the former computations, a conjugate heat conduction problem is solved, with an imposed temperature gradient. In the latter, computations are performed only in the pore space, assuming a no-slip boundary condition on the metal-pore space interface. For the thermal conductivity computation, given temperatures are imposed on the two opposing faces of the cuboidal domain, while the lateral boundaries are assumed to be adiabatic; the heat conduction equation is solved in both the fluid and solid regions, and a continuity of temperature and heat flux is imposed at the interface. For the flow problem, a pressure-outlet boundary condition is employed in conjunction with a velocity inlet boundary condition on opposite boundaries, while the lateral boundaries are assumed to be symmetry boundaries. Although a fully developed flow assumption is not made, we found that the cross-sectionally averaged pressure achieved a linear profile within the sample for all the Reynolds numbers considered here. For computation of the Nusselt number, conduction through the metal is neglected and a constant heat flux boundary condition is imposed on the interface separating the pore space from the metal. As with the pressure drop computation, we did not explicitly impose a fully developed thermal condition; nevertheless, the Nusselt number was found to become constant in the flow direction for the sample sizes and flow conditions considered here. The boundary conditions for the 

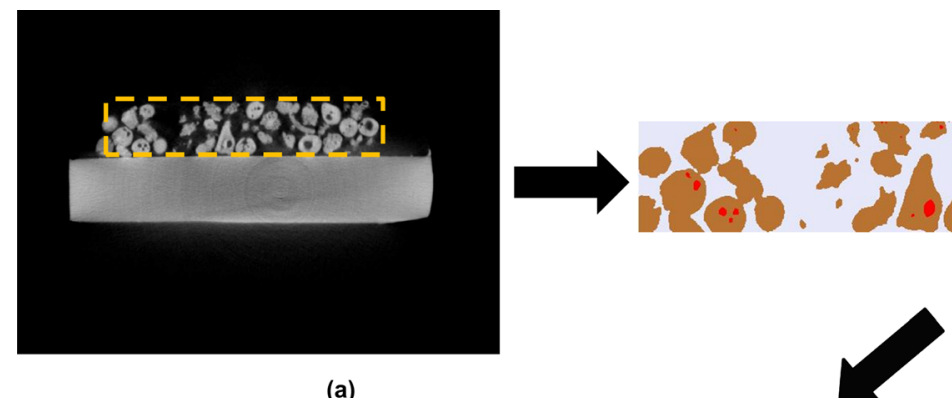

(a)

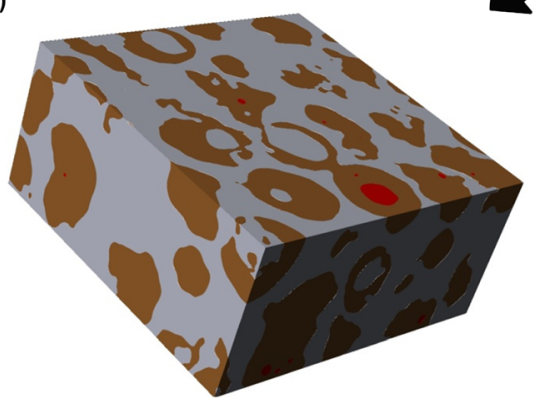

(b)

(c)

Fig. 2 Image processing and meshing showing (a) original image with region of interest identified, (b) segmented image, and (c) image after surface/volume reconstruction. (The red regions indicate voids in the particles.)

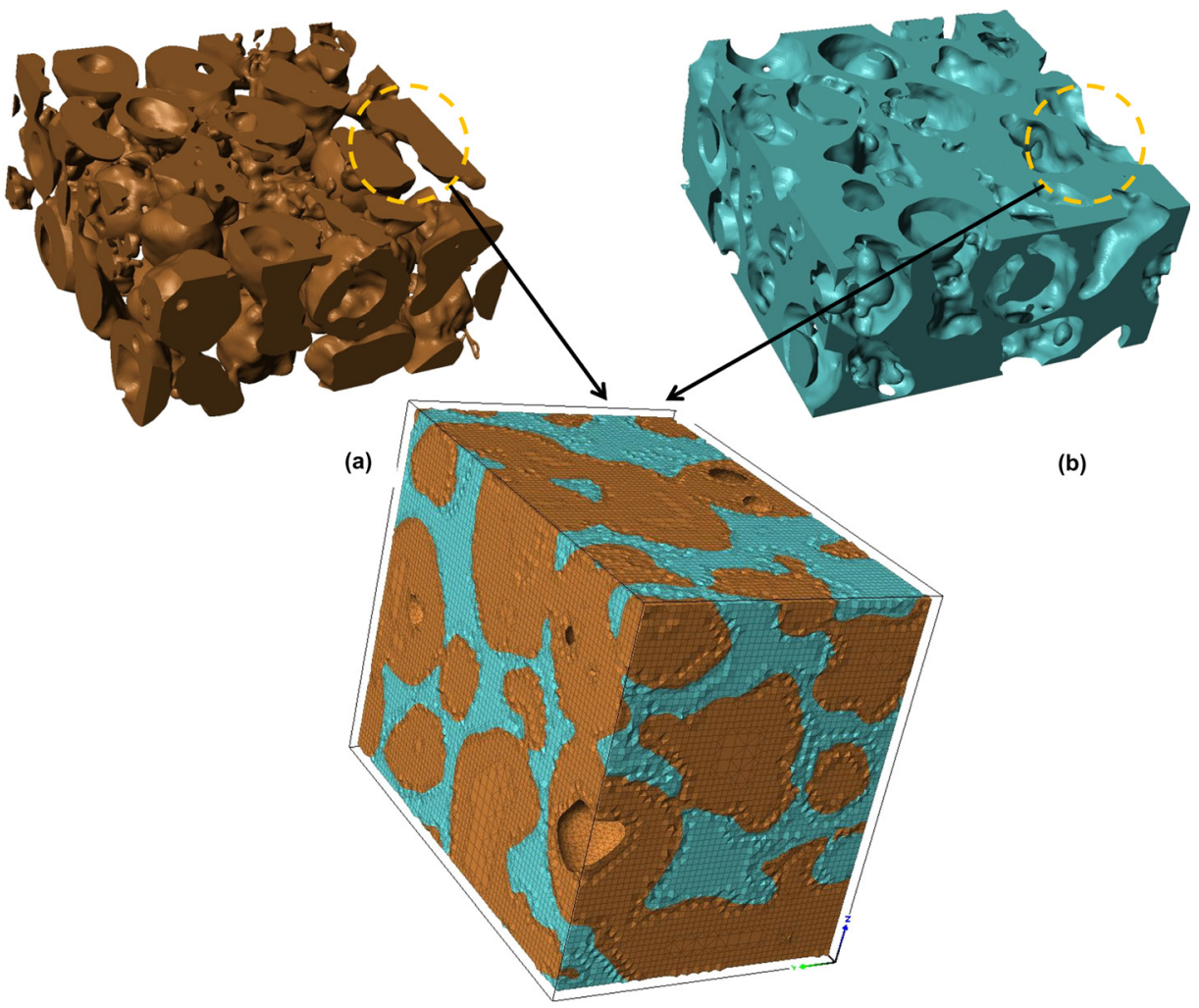

Fig. 3 (a) Sintered copper and (b) surrounding pore space. An inset of mesh for a 250-355 $\mu \mathrm{m}$ sample is also shown.

two types of computations are shown in Figs. $4(a)$ and $4(b)$, respectively.

3.2 Solution Procedure. For the domain sizes in this work, computational meshes of approximately $20 \times 10^{6}$ cells are generated using the procedure described in the previous section. The governing equations are solved using the commercial CFD solver FLUENT [20], employing a second-order upwind scheme for the flow and heat transfer calculations. Pressure-velocity coupling is addressed using the SIMPLE algorithm, along with an algebraic multigrid algorithm for solving the linearized system of governing equations. Details of the numerical method may be found in Ref. [20]. Also, the governing equations are suitably under-relaxed to 


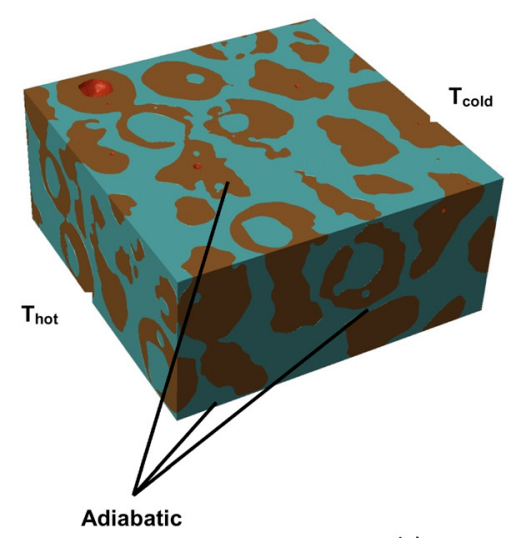

(a)

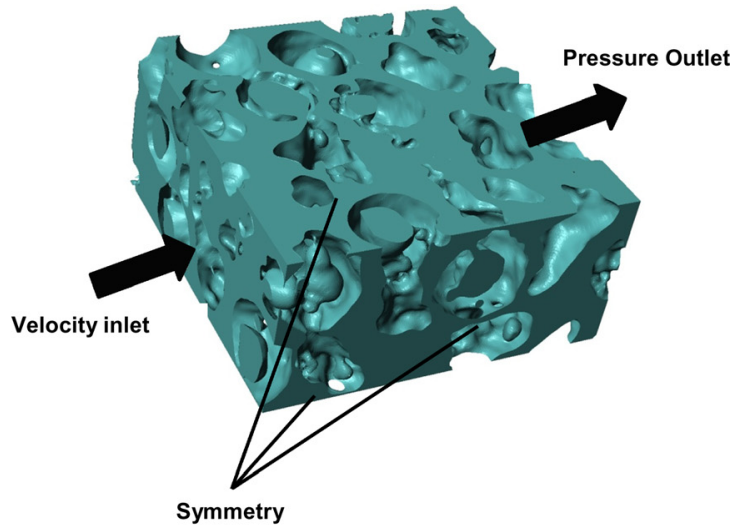

(b)

Fig. 4 Boundary conditions for (a) conjugate conduction problem and (b) flow problem

Table 1 Porosity, average effective thermal conductivity and necking ratio predictions

\begin{tabular}{|c|c|c|c|c|c|c|c|}
\hline Sample & $\begin{array}{c}\text { Porosity, } \varepsilon \\
\text { (nominal) }\end{array}$ & $\begin{array}{l}\text { Porosity, } \varepsilon \\
\text { (measured) }\end{array}$ & $\begin{array}{c}k_{\text {eff, air }} \\
(\mathrm{W} / \mathrm{m} \cdot \mathrm{K})\end{array}$ & $\begin{array}{l}\text { Standard deviation } \\
\text { of } k_{\text {eff, }} \text { air }\end{array}$ & $\begin{array}{l}k_{\text {eff, water }} \\
(\mathrm{W} / \mathrm{m} \cdot \mathrm{K})\end{array}$ & $\begin{array}{c}\text { Standard deviation } \\
\text { of } k_{\text {eff, water }}\end{array}$ & $\begin{array}{l}\text { Necking ratio, } \\
\mathrm{Nr}\left(\mathrm{mm}^{-1}\right)\end{array}$ \\
\hline $45-75 \mu \mathrm{m}$ (a) & 0.61 & $0.61-0.63$ & 53.96 & 3.98 & 55.66 & 3.94 & 1.93 \\
\hline $106-150 \mu \mathrm{m}(\mathrm{b})$ & 0.64 & $0.62-0.65$ & 28.46 & 4.22 & 35.49 & 3.89 & 1.43 \\
\hline $250-355 \mu \mathrm{m}(\mathrm{c})$ & 0.63 & $0.60-0.63$ & 13.83 & 3.24 & 17.91 & 3.17 & 1.06 \\
\hline
\end{tabular}

ensure proper convergence. The default convergence criterion in FLUENT, based on the scaled residuals, is employed for termination. For the heat transfer computations, the flow equations are decoupled from the energy equation and hence the continuity and momentum equations are first solved to convergence. After this, the energy equation is solved to convergence, with the converged flow solution assumed fixed.

\section{Results and Discussion}

The flow and thermal transport through sintered copper microstructures is modeled by considering three samples with varying particle-size ranges: (a) 45-75 $\mu \mathrm{m}$, (b) 106-150 $\mu \mathrm{m}$, and (c) $250-355 \mu \mathrm{m}$. The porosity of the samples as provided by the manufacturer (Thermacore, Inc.) is 61, 64, and 63\%, respectively (measured porosities are provided in Table 1). As described previously, three to four subsamples are considered for each particlesize range to statistically average out variations in the predicted parameters. Typical computational domain sizes are $1.5 \mathrm{~mm} \times 0.5$ $\mathrm{mm} \times 1.5 \mathrm{~mm}$, each for the lowest and intermediate particle sizes, and $3.8 \mathrm{~mm} \times 0.7 \mathrm{~mm} \times 1.5 \mathrm{~mm}$ for sample with the biggest particle size. Based on the scanned sample sizes and number of particles per subsample, the smallest particle-size sample is subdivided into three, while the intermediate and largest particlesize samples are subdivided into four subsamples. The computed parameters include: porosity, effective thermal conductivity, permeability, Ergun's coefficient, and Nusselt number for interfacial heat transfer.

4.1 Effective Thermal Conductivity and Necking Ratio. The effective thermal conductivity is computed for dry and water-saturated samples. For the dry case, the samples are assumed to be saturated with air. As noted earlier, some of the particles were found to be hollow; for solution of the conjugate conduction problem, the void space in the particles is assumed to be filled with air for both the dry and water-saturated cases. For computing effective thermal conductivity in a direction $x_{i}$, a temperature gradient $\left(\frac{\partial T}{\partial x_{i}}\right)$ is imposed across the two ends of the cuboidal sample, holding the lateral faces adiabatic. No pressure gradient is imposed, and fluid in the pore space is considered static. The effective thermal conductivity, $k_{e f f}$, is calculated as Ref. [21]

$$
k_{\text {eff }}=\frac{-\int \mathbf{J} \cdot d \mathbf{A}}{\left(\frac{\partial T}{\partial x_{i}}\right) A}
$$

$\boldsymbol{J}$ is the heat flux vector and $d \boldsymbol{A}$ is the outward pointing area vector. The effective thermal conductivity is calculated by considering conduction through both the solid and fluid regions, i.e., the integral in Eq. (4) is performed on both the solid and fluid regions of the face, and $A$ is the total conduction area perpendicular to direction $x_{i}$. Temperature contours for a subsample of sample $(c)$ are shown in Fig. 5 for a temperature difference of $100 \mathrm{~K}$ imposed across the sample.

The effective thermal conductivity was observed to be different along different directions, as also noted in our previous work [17]. Differences as high as $15-20 \%$ were observed for the same subsample along different directions. This anisotropy is attributed to the large difference in the number of particles along different directions; for the samples considered here, there were as few as three to seven particles along the smallest dimension, while the particles along the longer direction number 15-35. Furthermore, as noted in our previous work [17], we considered multiple realizations to mitigate some of the domain size artifacts. The effective thermal conductivity data, averaged over the three directions and various subsamples, along with the nominal porosity, measured porosity, and the observed standard deviation, is presented in Table 1. The standard deviation is measured with respect to the subsamples, i.e., after averaging out the effective thermal conductivity values over the three directions. The computations show that in contrast to other porous media such as metal foams $[17,21]$, the effective thermal conductivity for sintered microstructures is a strong function of the microstructure. As can be noted from Table 1, the effective thermal conductivity for the three samples is drastically different despite the similarity in their porosities. To further characterize this difference, an average necking ratio, $N r$, is calculated as 


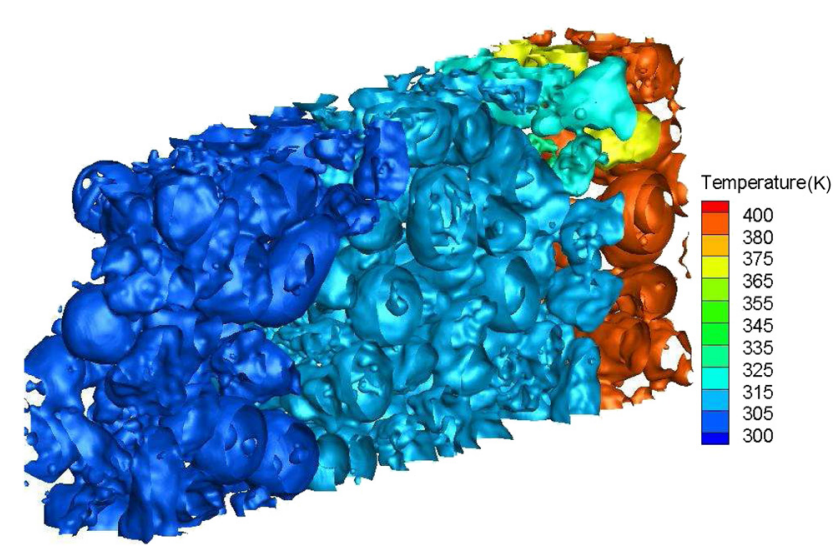

Fig. 5 Temperature contours for conduction in a 250-355 $\mu \mathrm{m}$ subsample

$$
N r=\frac{A_{\text {neck }}}{V_{T}}, A_{\text {neck }}=\frac{A_{s f, \text { segmented }}-A_{s f}}{2}
$$

Here, $A_{s f}$ is the solid-pore interfacial area, while $A_{s f \text {,segmented }}$ is the total surface area of all the particles after segmentation, i.e., after identifying each particle individually, as described in our previous work [22], employing the software package Avizo [23]. The presintered packed bed of particles has essentially zero interparticle contact area, while, as sintering proceeds, particle-particle contact area increases. The parameter, $\mathrm{Nr}$, characterizes the amount of sintering through the measurement of interparticle contact area per unit volume in the post sintered sample, which governs the effective thermal conductivity. The particles so identified for the case of a $250 \mu \mathrm{m}$ subsample are shown in Fig. 6. The results for the necking ratio are tabulated in Table 1 for the three samples considered. It may be observed that the most conductive sample demonstrates the highest necking ratio and vice versa. It is noted that the necking ratio as reported in this work is calculated for the largest dataset that could be analyzed under the constraints on memory and thus a single value of necking ratio is reported for each particle size; for meshing and postprocessing, each dataset is divided into three to four subvolumes.

The effective thermal conductivity is plotted in Fig. 7 as a function of necking ratio. It is clear that the effective thermal conductivity increases as the necking ratio increases. The main pathway for heat transfer is through the highly conducting solid matrix, and thus similar observations are made whether the matrix is satu-

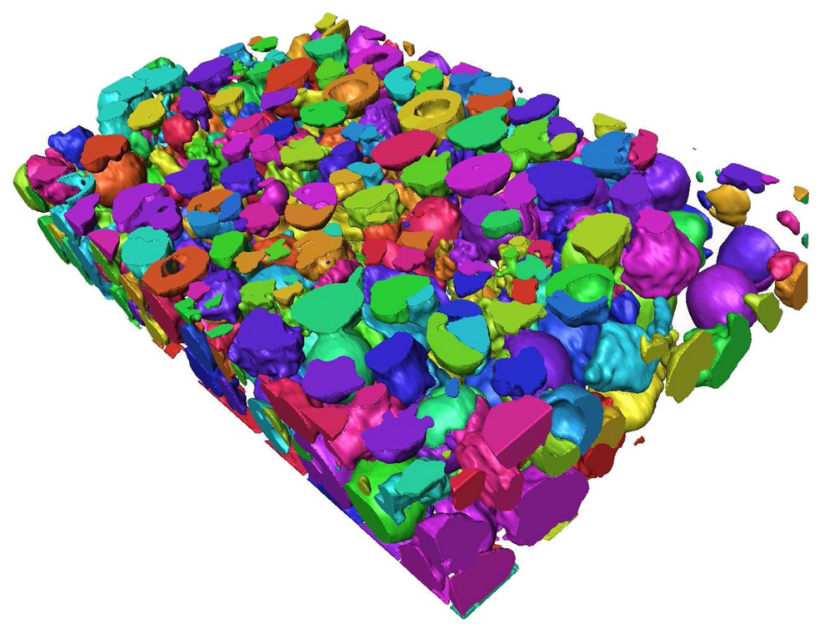

Fig. 6 Particles identified by color in a 250-355 $\mu \mathrm{m}$ subsample

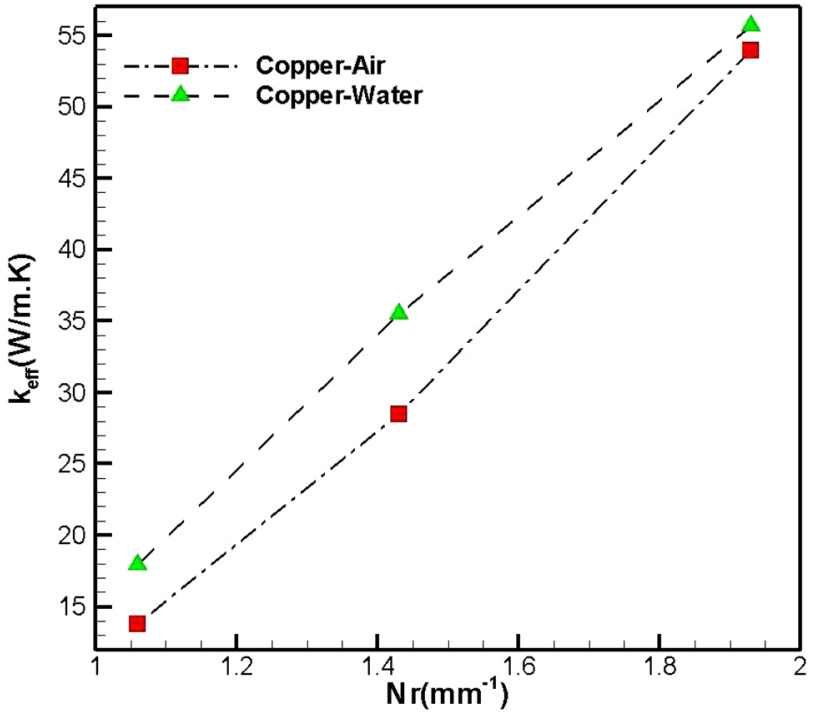

Fig. 7 Effective thermal conductivity as a function of necking ratio with air and water as the saturating liquids

rated with air or water. Figure 7 also suggests a nearly linear dependence of effective thermal conductivity on the necking ratio, which may be represented by the following relationship and be directly employed for the case of sintered copper samples with porosity in the range of $60 \%$ and saturated with air or water

$$
k_{\text {eff }}=a \times N r+b
$$

The values of the fitting parameters, $a$ and $b$, are provided in Table 2.

The effective thermal conductivity results are compared with analytical models available in the literature such as the MaxwellEucken models [7] and the effective medium theory (EMT) model $[8,9]$. The Maxwell-Eucken models consider two extreme cases: (a) conducting spherical particles suspended in a nonconducting matrix and (b) nonconducting spherical particles in a conducting matrix. Expressions for effective thermal conductivity are obtained by considering the separation distance between the particles to be large compared to the particle diameter, such that the solution to the temperature field may be obtained without considering the effect of neighboring particles, as also described by Carson et al. [10]. The effective thermal conductivity relations for these two cases are given by

$$
\begin{gathered}
k_{\text {eff }}=k_{1} \frac{2 k_{1}+k_{2}-2\left(k_{1}-k_{2}\right) \varepsilon}{2 k_{1}+k_{2}+\left(k_{1}-k_{2}\right) \varepsilon} \\
k_{\text {eff }}=k_{2} \frac{2 k_{2}+k_{1}-2\left(k_{2}-k_{1}\right)(1-\varepsilon)}{2 k_{2}+k_{1}+\left(k_{2}-k_{1}\right)(1-\varepsilon)}
\end{gathered}
$$

Here, $k_{1}$ and $k_{2}$ refer to the thermal conductivity of the surrounding matrix and of the suspended particles, respectively. Also, $\varepsilon$ refers to the porosity or the volume fraction of the continuous phase.

The second model used in the comparison is the EMT model, which considers the two phases, i.e., the conducting and the

Table 2 Fitting parameters for the proposed effective thermal conductivity correlation

\begin{tabular}{lcc}
\hline \hline Working fluid & $\mathrm{a}(\mathrm{W} / \mathrm{K})$ & $\mathrm{b}(\mathrm{W} / \mathrm{m} . \mathrm{K})$ \\
\hline Air & 46.4 & -36.28 \\
Water & 43.22 & -27.32 \\
\hline \hline
\end{tabular}




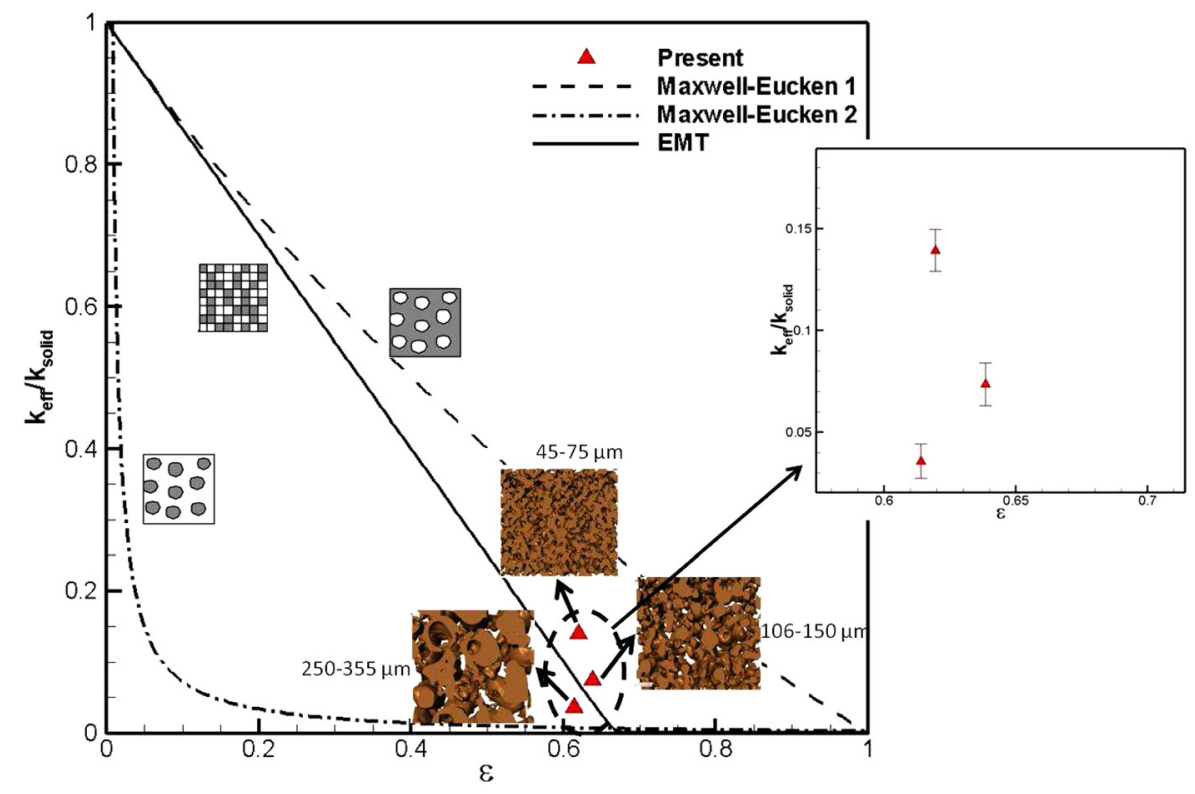

Fig. 8 Effective thermal conductivity as a function of porosity. In the present computations, $k_{\text {solid, }} \boldsymbol{k}_{\text {water }}$ and $\boldsymbol{k}_{\text {air }}$ are $387.5,0.613$, and $0.0265 \mathrm{~W} / \mathrm{m} . \mathrm{K}$, respectively.

nonconducting phases, to be randomly dispersed. The expression below is obtained from the Maxwell theory, with some modifications as explained in Ref. [10].

$$
\begin{aligned}
k_{\text {eff }}= & \frac{1}{4}\left((3 \varepsilon-1) k_{2}+[3(1-\varepsilon)-1] k_{1}\right. \\
& \left.+\sqrt{\left.(3 \varepsilon-1) k_{2}+(3\{1-\varepsilon\}-1) k_{1}\right]^{2}+8 k_{1} k_{2}}\right)
\end{aligned}
$$

A comparison of the present computations with the MaxwellEucken and EMT models is shown in Fig. 8, along with error bars in the inset. As described in Carson et al. [10], the effective conductivity of open-pore structures (such as granular porous media) lies between the lower Maxwell-Eucken model (Eq. (7)) and the EMT model, while that of closed-pore structures (such as closedcell foams) lies between the EMT and the higher Maxwell-Eucken model (Eq. (8)). The sintered samples considered here exhibit conductivities that fall below and above the EMT model predictions. Samples (a) and (b) were under-predicted by the EMT model by about $40 \%$, while for sample (c), the EMT model overpredicts by about $50 \%$. Nonetheless, it may still be concluded that the EMT model is better-suited for describing the effective thermal conductivity of sintered samples than the Maxwell-Eucken models that are conventionally employed [1].

4.2 Permeability, Ergun's Coefficient, and Friction Factor. Traditionally, flow through porous media is often analyzed by considering Darcy's law [13], which is an empirical relation between pressure drop and flow speed that is valid at low Reynolds numbers. At higher Reynolds numbers, the expression is modified to include nonlinear effects, as given by [13]

$$
\begin{gathered}
-\frac{\partial P}{\partial x_{i}}=\frac{\mu}{K} U_{\text {mod }}+\frac{\rho C_{E}}{\sqrt{K}} U_{\text {mod }}^{2} \\
U_{\text {mod }}=\frac{A_{f}}{A_{T}} U_{\text {in }}
\end{gathered}
$$

Here, $U_{\text {mod }}$ is a superficial velocity called Darcian velocity, obtained by considering the porous medium as a continuum and neglecting the pore-scale features, i.e., $A_{f}$ is the total area available for fluid flow at the inlet, i.e., the area of the pore space at the inlet, and $A_{T}$ is the total area of cross-section of the sintered block, i.e., the area of the metal and pore space combined (area of crosssection of the cuboidal sample at the inlet). $C_{E}$ and $K$ in Eq. (10) are the Ergun's coefficient and permeability, respectively. Equation (10) is a specific form of the Forchheimer equation, with a permeability-dependent Forchheimer coefficient. For this computation, as described previously, velocity inlet and pressure-outlet boundary conditions are employed in the flow direction. All the boundaries transverse to the main flow direction are made symmetric, and a no-slip boundary condition is employed at the solid-fluid interface. The resulting pressure drop is then used for computing the permeability and Ergun's coefficient. The results are reported by considering pressure drop in the fully developed region, identified as a region with linear pressure drop. The computations are performed for a variety of inlet speeds in the Reynolds number (based on permeability) range of $0-10$. Water is considered as the interstitial fluid for both flow and convective heat transfer computations.

Permeability and Ergun's coefficient are calculated as leastsquare fits to the variation of pressure drop with inlet speed. For this, the curve-fitting tool in MATLAB is employed, and the data are fit with second-order polynomials. The pressure drop variation with inlet flow speed for a representative subsample is shown in Fig. 9. As with the effective thermal conductivity results, the flow computations are also reported as values averaged over the subsamples. However, in contrast to the effective thermal conductivity, the samples are found to be isotropic with respect to permeability. Also, the smallest dimension which has only three to seven particles is not considered for flow computations, for lack of sufficient length for the flow to become fully developed. Hence, all the results pertaining to flow and interfacial heat transfer are based on the longer dimensions.

Table 3 shows the permeability and Ergun's coefficient data for the three particle sizes considered in this work. It may be observed that permeability is a direct function of particle (pore) size: the sample with the highest particle size exhibits the highest permeability. Also shown in Table 3 are the standard deviations of the computed values and the permeabilities obtained using the Carman-Kozeny equation $[1,14]$

$$
K=\frac{D_{p}^{2} \varepsilon^{3}}{150(1-\varepsilon)^{2}}
$$




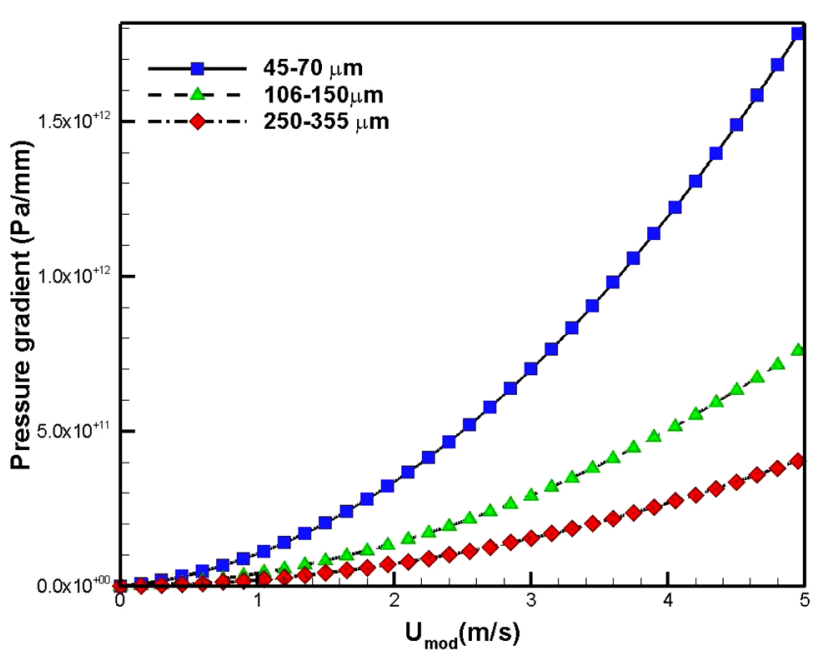

Fig. 9 Pressure drop variation with modified inlet velocity. Representative values for a subsample for each of the three samples considered in this work are shown.

Table 3 Permeability and Ergun's coefficient predictions

\begin{tabular}{lccccc}
\hline \hline Sample & $\mathrm{K}\left(\mathrm{m}^{2}\right)$ & $\begin{array}{c}\text { Standard } \\
\text { deviation } \\
\text { of K }\end{array}$ & $\begin{array}{c}\mathrm{K} \text { from } \\
\text { Eq. }(12) \\
\left(\mathrm{m}^{2}\right)\end{array}$ & $C_{E}$ & $\begin{array}{c}D_{p} \\
(\mu \mathrm{m})\end{array}$ \\
\hline $45-75 \mu \mathrm{m} \mathrm{(a)}$ & $2.51 \times 10^{-11}$ & $4.30 \times 10^{-12}$ & $6.44 \times 10^{-11}$ & 0.266 & 74.3 \\
$106-150 \mu \mathrm{m}(\mathrm{b})$ & $7.89 \times 10^{-11}$ & $1.54 \times 10^{-11}$ & $3.46 \times 10^{-10}$ & 0.267 & 102 \\
$250-355 \mu \mathrm{m}(\mathrm{c})$ & $2.63 \times 10^{-10}$ & $5.15 \times 10^{-11}$ & $1.56 \times 10^{-09}$ & 0.249 & 215 \\
\hline
\end{tabular}

Equation (12) is a semi-empirical relation, obtained by considering unconsolidated packed, spherical particles in a square packed configuration. Here $D_{p}$ is the effective particle diameter defined as

$$
D_{p}=\frac{6 V_{s}}{A_{s f}}
$$

The results obtained by using this correlation are on average higher by a factor of 3 than the present computations. This is attributed to the nonspherical particles as well as the semiconsolidated state of the porous media samples considered, as can be noted from Fig. 3 .

In addition to permeability and Ergun's coefficient, the friction factor $f$ is also estimated. The present results are compared with results obtained by considering the modified Ergun's equation for pressure drop given by Macdonald et al. [12] for smooth spherical particles [14]

$$
-\frac{\partial P}{\partial x_{i}}=\frac{\mu}{K} U_{m o d}+1.8 \frac{1-\varepsilon}{\varepsilon^{3}} \frac{\rho U_{m o d}^{2}}{D_{p}}
$$

At a given Reynolds number, friction factor is defined as in Ref. [21]

$$
f=\frac{\left(-\frac{\partial P}{\partial x_{i}}\right) \sqrt{K}}{\rho U_{\text {mod }}^{2}}
$$

$\left(-\frac{\partial P}{\partial x_{j}}\right)$ is the imposed pressure gradient in direction $x_{i}$. It may be noted that our definition of friction factor along with the definition of permeability leads to an alternate expression for the friction factor

$$
f=\frac{1}{R e_{K}}+C_{E}
$$

$$
R e_{K}=\frac{\rho U_{\text {mod }} \sqrt{K}}{\mu}
$$

The friction factor data are compared with that from Ergun's equation (Eq. (14)), which is given as

$$
f=\frac{1}{R e_{K}}+C_{E}^{\prime}
$$

Here, $C_{E}$ ' is the equivalent Ergun's coefficient from Eq. (14), i.e.,

$$
C_{E}^{\prime}=1.8 \frac{1-\varepsilon}{\varepsilon^{2}} \frac{\sqrt{K}}{D_{p}}
$$

Figure 10 shows the friction factor results for the three sintered samples considered in this work. As with the other properties, friction factor is also averaged over the multiple subsamples. It may be observed from Fig. 9 that at smaller velocities (Reynolds numbers), pressure drop is a linear function of velocity and hence the slope in Fig. 10 is linear. However, at higher Reynolds numbers, the second, nonlinear term in Eq. (10) dominates and contributes significantly to the overall pressure drop, and the present results deviate from the correlation.

4.3 Interfacial Heat Transfer. The heat transfer coefficient is now estimated for the case of thermally fully developed flow, which in general could be obtained for either a constant temperature or a constant heat flux wall boundary condition. With laminar flow, the Nusselt number based on a constant temperature boundary condition is expected to be up to $10-15 \%$ different from that with a constant heat flux boundary condition. Therefore, in the current study, the heat transfer coefficient is estimated by imposing a constant heat flux boundary condition, the resulting equations for which are easier to solve compared to the constant temperature case. A constant heat flux boundary condition is imposed at the solid-fluid interface and the domain boundaries in the transverse direction are considered adiabatic. As noted earlier, since the flow field is decoupled from the energy equation, we begin the heat transfer computations with the converged flow field solution. Once the energy equation is also converged, interfacial heat transfer coefficient is calculated as defined below

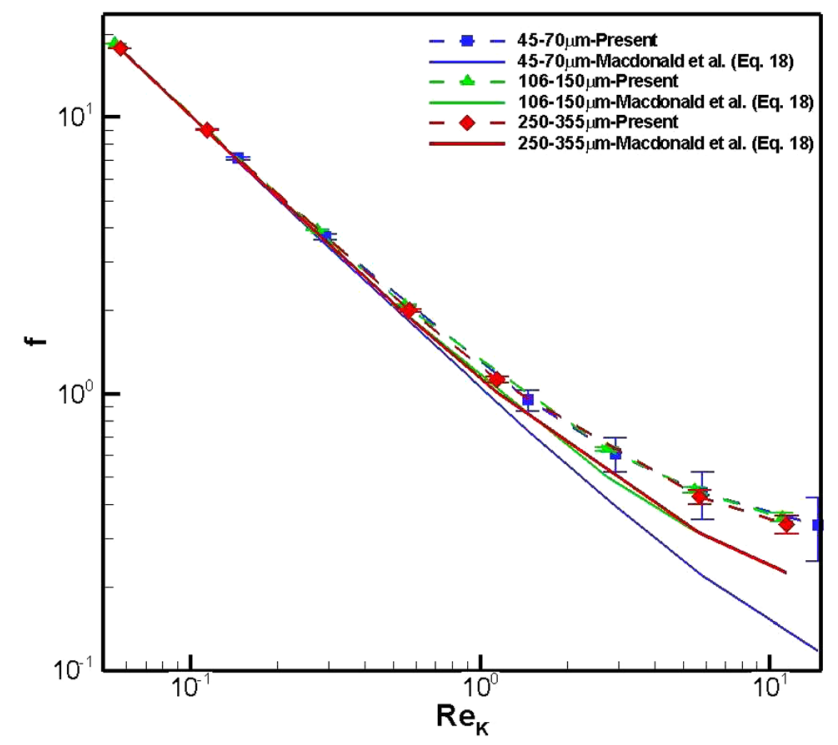

Fig. 10 Variation of friction factor with Reynolds number. The present calculations are compared with results from the literature [12]. 


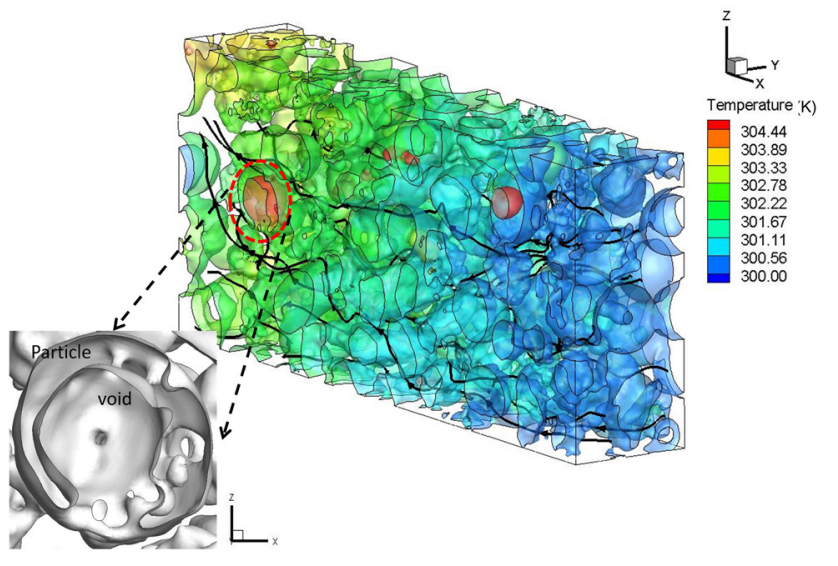

Fig. 11 Representative streamtraces and temperature contours in a 250-355 $\mu \mathrm{m}$ subsample for forced convection through the sintered particle bed. An inset of the punctured particle is also shown.

$$
h_{s f}=\frac{q^{\prime \prime}}{\left(\overline{T_{s f}}-\overline{T_{f}}\right)}
$$

$\overline{T_{s f}}$ is the average temperature of the solid-fluid interface, $\overline{T_{f}}$ is the average fluid temperature in the pore region, and $q^{\prime \prime}$ is the imposed heat flux. Figure 11 shows streamtraces through a $250-355 \mu \mathrm{m}$ subsample along with temperature contours. It may be noted that the hot and cold "islands" in Fig. 11 correspond to the punctured particles as shown in the inset. These particles have a re-entrant pore which is poorly connected to the bulk pore space. Since the heat flux boundary condition is applied to the solid-fluid interface inside these particles, a local high temperature results because of the lack of sufficient fluid flow inside these re-entrant spaces. The computed Nusselt number is compared with a correlation for Nusselt number proposed by Wakao and Kaguei [15], who compiled experimental results for both steady and transient experiments of interfacial heat transfer for flow through spherical particle beds, as noted by Kaviany [14]. The correlation is given as

$$
N u_{s f}=\frac{h_{s f} D_{p}}{k}=2+1.1 \operatorname{Re}_{D_{p}}^{0.6} \operatorname{Pr}^{1 / 3}
$$

where $R e_{D p}$ is the particle (pore) Reynolds number defined as

$$
\operatorname{Re}_{D_{p}}=\frac{\rho U_{m o d} D_{p}}{\mu}
$$

$D_{p}$ is the effective particle diameter, given by Eq. (13). The results for interfacial Nusselt number, along with the Wakao and Kaguei correlation (Eq. (21)), are plotted in Fig. 12. It may be observed from Fig. 12 that the Wakao and Kaguei correlation, developed for spherical particle beds, agrees qualitatively with the computations but does not provide accurate quantitative predictions of the interfacial heat transfer through sintered beds. Also important to note is the deviation of the correlation with respect to the experimental data on which it was based, as discussed in Ref. [14], and the lack of data in the near-zero-Reynolds number range.

In view of these limitations of the Wakao and Kaguei correlation, we propose a new correlation valid for sintered beds (with porosity in the range of $60 \%$ ), obtained for the range of Reynolds numbers considered in this work

$$
N u_{s f}=a+b\left(\operatorname{Re}_{D_{p}}^{0.6} \operatorname{Pr}^{1 / 3}\right)^{1.2}
$$

The proposed correlation is also plotted in Fig. 12. The coefficients in Eq. (22) are tabulated in Table 4 for the three samples

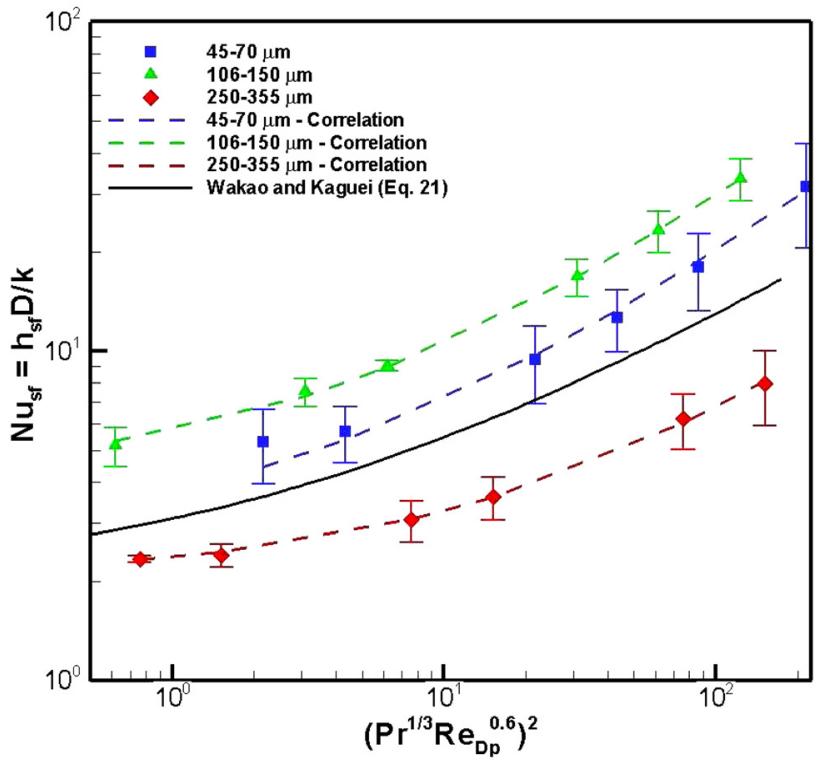

Fig. 12 Nusselt number as a function of Reynolds number. The present computations and proposed correlations are compared with the Wakao and Kaguei correlation (Eq. (21)) [15].

Table 4 Fitting parameters for the proposed interfacial heat transfer correlation

\begin{tabular}{lcc}
\hline \hline Sample & $\mathrm{a}$ & $\mathrm{b}$ \\
\hline $45-75 \mu \mathrm{m} \mathrm{(a)}$ & 2.687 & 1.110 \\
$106-150 \mu \mathrm{m} \mathrm{(b)}$ & 4.133 & 1.616 \\
$250-355 \mu \mathrm{m} \mathrm{(c)}$ & 2.081 & 0.296 \\
\hline \hline
\end{tabular}

under consideration. The R-squared values for the fitted curves are $0.994,0.999$, and 0.998 for the $45-75 \mu \mathrm{m}, 106-150 \mu \mathrm{m}$, and 250-355 $\mu \mathrm{m}$ samples, respectively.

\section{Conclusions}

Accurate single-phase heat transfer characterization of monoporous sintered copper, employed as wicks in commercial heat pipes, is performed using numerical modeling based on geometrically faithful meshes generated through $\mu$-CT scanning. Three samples of varying particle-size ranges but of nearly identical porosities are considered, and important single-phase heat transfer parameters such as effective thermal conductivity, permeability, and interfacial Nusselt number are estimated. Through the present simulations, the dependence of the effective thermal conductivity of sintered samples on parameters other than porosity, such as necking ratio, is demonstrated and the need for robust correlations taking into effect such dependencies is highlighted. The present computations are compared with some of the popular models employed by heat pipe designers for predicting device-level performance. It is observed that the most widely used models, viz., the Maxwell-Eucken models, over-/under-predict the effective thermal conductivity of the present samples by significant amounts. Furthermore, the results are compared with the EMT model, and it is demonstrated that as a first-order approximation, the EMT model can be used to predict the effective thermal conductivity of sintered samples.

Permeability and Ergun's coefficient are estimated for low Reynolds number flow through the sintered bed, employing Darcy's law. The results obtained for permeability are compared with the widely employed Carman-Kozeny correlation (Eq. (12)). It is observed that Carman-Kozeny correlation, a semiheuristic 
model originally developed for packed beds of spherical particles, over-predicts the permeability of sintered beds. Ergun's coefficient and friction factor data are compared by considering a modified form of Ergun's equation developed by Macdonald et al. (Eq. (14)) [12]. Again, it is observed that the model, developed for smooth spherical particles, fails to accurately predict flow through sintered porous media, especially at higher Reynolds numbers, when the inertial contribution to pressure drop dominates the viscous part. Also, the equivalent Ergun's coefficient values employed in the modified Ergun equation, from Ref. [14], were found to be lower than those obtained from the current computations.

The interfacial Nusselt numbers obtained are compared with a correlation for spherical particle beds developed by Wakao and Kaguei (Eq. (21)) based on experimental data corresponding to the intermediate range of Reynolds numbers. It is observed that the correlation predicts the qualitative behavior fairly well, but the quantitative values differ from the current computations. The mismatch is attributed to a number of factors pointed out by Kaviany [14], such as lack of data in the low range of Reynolds numbers and the spread in the experimental data on which the correlation was based. To better predict the Nusselt number for sintered materials, correlations based on the current results are proposed for the three samples considered in this work.

\section{Nomenclature}

$A=$ base area

$a, b=$ fitting coefficients

$C=$ specific heat

$C_{E}, C_{E}^{\prime}=$ Ergun's coefficient

$D_{p}=$ effective particle diameter

$f=$ friction factor

$h_{s f}=$ interfacial heat transfer coefficient

$\vec{J}=$ diffusion flux vector

$k=$ thermal conductivity

$K=$ permeability

$N r=$ necking ratio

$\mathrm{Nu}=$ Nusselt number

$P=$ pressure

$\operatorname{Pr}=$ Prandtl number

$q^{\prime \prime}=$ imposed heat flux

$R e=$ Reynolds number

$T=$ temperature

$u_{i}=$ pore velocity in coordinate direction

$U_{\text {mod }}=$ modified velocity

$V_{s}=$ volume of solid

$x_{i}=$ Cartesian coordinate direction

\section{Greek Symbols}

$\varepsilon=$ porosity

$\mu=$ dynamic viscosity

$\rho=$ density

\section{Subscripts}

1,2 = matrix and suspended particles

air $=$ air in the pore region

$$
\text { eff }=\text { effective }
$$

$f=$ pore

in $=$ inlet

neck $=$ sintered neck

$s f=$ solid-fluid interface

solid $=$ sintered material

$T=$ total, solid and fluid

water $=$ water in the pore region

\section{References}

[1] Faghri, A., 1995, Heat Pipe Science and Technology, Taylor \& Francis, Washington DC

[2] Dunn, P. D., and Reay, D. A., 1973, “The heat pipe,” Phys. Technol., 4, p. 187.

[3] Garimella S. V., Joshi Y. K., Bar-Cohen A., Mahajan R., Toh, K. C., Carey, V. P., Baelmans, M., Lohan, J., Sammakia, B., and Andros, F., 2003, "Thermal Challenges in Next Generation Electronic Systems-Summary of panel Presentations and Discussions," IEEE Trans. Compon. Packag. Technol., 25(4), pp. $569-575$.

[4] Weibel, J. A., Garimella, S. V., and North, M. T., 2010, "Characterization of Evaporation and Boiling from Sintered Powder Wicks Fed by Capillary Action," Int. J. Heat Mass Transfer, 53, pp. 4204-4215.

[5] Dullien, F. A. L., 1979, Porous Media, Fluid Transport and Pore Structure, Academic, New York.

[6] Lin, Y. Y., Semenic, T., and Catton, I., 2005, "Thermophysical Properties of Monoporous Sintered Copper," Summer Heat Transfer Conference, ASME, New York, CA, pp. 17-23.

[7] Hashin, Z., and Shtrikman, S., 2009, "A Variational Approach to the Theory of the Effective Magnetic Permeability of Multiphase Materials," J. Appl. Phys. 33(10), pp. 3125-3131.

[8] Landauer, R., 2009, "The Electrical Resistance of Binary Metallic Mixtures," J. Appl. Phys., 23(7), pp. 779-784.

[9] Kirkpatrick, S., 1973, "Percolation and Conduction," Rev. Modern Phys., 45(4), pp. 574-588.

[10] Carson, J. K., Lovatt, S. J., Tanner, D. J., and Cleland, A. C., 2005, "Thermal Conductivity Bounds for Isotropic, Porous Materials," Int. J. Heat Mass Transfer, 48(11), pp. 2150-2158.

[11] Ergun, S., and Orning, A. A., 1949, "Fluid Flow Through Randomly Packed Columns and Fluidized Beds," Ind. Eng. Chem., 41(6), pp. 1179-1184.

[12] Macdonald, I. F., El-Sayed, M. S., Mow, K., and Dullien, F. A. L., 1979, "Flow Through Porous Media-The Ergun Equation Revisited," Ind. Eng. Chem. Fundam., 18(3), pp. 199-208.

[13] Vafai, K., 2005, Handbook of Porous Media, CRC Press, Boca Raton, FL, p. 41 .

[14] Kaviany, M., 2002, Principles of Heat Transfer, Wiley-Interscience, New York.

[15] Wakao, N., and Kagei, S., 1982, Heat and Mass Transfer in Packed Beds, Gordon and Breach Science, New York.

[16] Ranjan, R., Murthy, J. Y., and Garimella, S. V., 2009, "Analysis of the Wicking and Thin-Film Evaporation Characteristics of Microstructures," J. Heat Transfer, 131, p. 101001.

[17] Bodla K. K., Murthy J. Y., and Garimella S. V., 2010, "MicrotomographyBased Simulation of Transport through Open-Cell Metal Foams," Numer. Heat Transfer, Part A: Appl., 58(7), pp. 527-544.

[18] Tabor, G., Young, P. G., West, T. B., and Benattayallah, A., 2007, "Mesh Construction from Medical Imaging for Multiphysics Simulation: Heat Transfer and Fluid Flow in Complex Geometries," Eng. Appl. Comput. Fluid Mech. 1(2), pp. 126-135.

[19] ScanIP, ScanFE, and ScanCAD Tutorial Guide for simpleware 3.1, Simpleware Ltd., Exeter, UK, 2009

[20] Users Guide for FLUENT 6.0, Fluent Inc., 2002.

[21] Krishnan, S., Murthy, J. Y., and Garimella, S. V., 2006, "Direct Simulation of Transport in Open-Cell Metal Foams," J. Heat Transfer, 128(8), pp. 793-799.

[22] Bodla, K. K., Murthy, J. Y., and Garimella, S. V., 2010, "Resistance NetworkBased Thermal Conductivity Model for Metal Foams," Comput. Mater. Sci. 50(2), pp. 622-632.

[23] User's Guide for AVIZO 6.2, 2009. 